PROCEEDINGS OF THE

AMERICAN MATHEMATICAL SOCIETY

Volume 134, Number 8, Pages 2205-2212

S 0002-9939(06)08427-9

Article electronically published on March 21, 2006

\title{
FREE MODULES OF RELATIVE INVARIANTS AND SOME RINGS OF INVARIANTS THAT ARE COHEN-MACAULAY
}

\author{
LARRY SMITH
}

(Communicated by Bernd Ulrich)

\begin{abstract}
Let $\rho: G \hookrightarrow \operatorname{GL}(n, \mathbb{F})$ be a faithful representation of a finite group $G$ and $\chi: G \longrightarrow \mathbb{F}^{\times}$a linear character. We study the module $\mathbb{F}[V]_{\chi}^{G}$ of $\chi$ relative invariants. We prove a modular analogue of result of R. P. Stanley and $\mathrm{V}$. Reiner in the case of nonmodular reflection groups to the effect that these modules are free on a single generator over the ring of invariants $\mathbb{F}[V]^{G}$. This result is then applied to show that the ring of invariants for $H=\operatorname{ker}(\chi) \leq G$ is Cohen-Macaulay. Since the Cohen-Macaulay property is not an issue in the nonmodular case (it is a consequence of a theorem of Eagon and Hochster), this would seem to be a new way to verify the Cohen-Macaulay property for modular rings of invariants. It is known that the Cohen-Macaulay property is inherited when passing from the ring of invariants of $G$ to that of a pointwise stabilizer $G_{U}$ of a subspace $U \leq V=\mathbb{F}^{n}$. In a similar vein, we introduce for a subspace $U \leq V$ the subgroup $G_{\langle U\rangle}$ of elements of $G$ having $U$ as an eigenspace, and prove that $\mathbb{F}[V]^{G}$ Cohen-Macaulay implies $\mathbb{F}[V]^{G}\langle U\rangle$ is also.
\end{abstract}

Fix a ground field $\mathbb{F}$ and let $V=\mathbb{F}^{n}$ be the $n$-dimensional vector space over $\mathbb{F}$ on which the group $\operatorname{GL}(n, \mathbb{F})$ of $n \times n$ invertible matrices over $\mathbb{F}$ acts. This note is concerned with conditions under which the ring of invariants of a finite subgroup of $\operatorname{GL}(n, \mathbb{F})$ is Cohen-Macaulay. In the nonmodular case the Cohen-Macaulay property is not an issue: It always holds as a consequence of a result of Eagon and Hochster (see, e.g., [5], Proposition 6.7.8).

Recent work [3] of V. Reiner, D. Stanton, and P. Webb have focused attention on the invariant theory of pairs of finite subgroups $H \triangleleft G \leq \operatorname{GL}(n, \mathbb{F})$, where $G$ is a subgroup of the normalizer $N_{\mathrm{GL}(n, \mathbb{F})}(H)$ of $H$ in $\mathrm{GL}(n, \mathbb{F})$. In particular, for their purposes, it becomes important to know how properties of $\mathbb{F}[V]^{G}$ are reflected in properties of $\mathbb{F}[V]^{H}$. Specifically, in constructing examples to illustrate some of the results of their paper, they were led to pose the following problem.

Problem (V. Reiner, D. Stanton and P. Webb). Let $\rho: H \hookrightarrow \operatorname{GL}(n, \mathbb{F})$ be $a$ representation of a finite group $H$ over the field $\mathbb{F}$ and let $G \leq \mathbb{N}_{\mathrm{GL}(n, \mathbb{F})}(H)$ be a subgroup of the normalizer of $\rho(H)$ in $\mathrm{GL}(n, \mathbb{F})$. If $\mathbb{F}[V]^{G}$ is a polynomial algebra, then is $\mathbb{F}[V]^{H}$ Cohen-Macaulay?

One purpose of this note is to prove the following result which was suggested by the examples of 3 .

Received by the editors March 4, 2005

2000 Mathematics Subject Classification. Primary 13A50, 13 C14.

(C)2006 American Mathematical Society Reverts to public domain 28 years from publication 
Theorem. Let $\mathbb{F}$ be a field, $n \in \mathbb{N}$ a natural number, and $H \leq \mathrm{GL}(n, \mathbb{F})$ a finite subgroup. Suppose that $G$ is a finite subgroup of the normalizer $N_{\mathrm{GL}(n, \mathbb{F})}(H)$ of $H$ in $\operatorname{GL}(n, \mathbb{F})$ such that $\mathbb{F}[V]^{G}$ is a polynomial algebra. If $G / H$ is cyclic of order prime to the characteristic of $\mathbb{F}$, then $\mathbb{F}[V]^{H}$ is Cohen-Macaulay.

It is known that the Cohen-Macaulay property is inherited when passing from the ring of invariants of $G$ to that of a pointwise stabilizer $G_{U}$ of a subspace $U \leq$ $V=\mathbb{F}^{n}$ (see, e.g., [6]). We introduce a related subgroup $G_{\langle U\rangle}$ consisting of those elements of $G$ having $U$ as an eigenspace, and prove $G_{\langle U\rangle}$ has a similar property.

Theorem. Let $\rho: G \hookrightarrow \operatorname{GL}(n, \mathbb{F})$ be a representation of a finite group over the field $\mathbb{F}$ and let $U \leq V=\mathbb{F}^{n}$ be a subspace. If $\mathbb{F}[V]^{G}$ is Cohen-Macaulay, then so is $\mathbb{F}[V]^{G\langle U\rangle}$.

As hindsight has shown, it is actually more convenient to set things up in a slightly different way, one which allows us to relate the Cohen-Macaulay property of $\mathbb{F}[V]^{H}$ to freeness properties of the modules of relative invariants $\mathbb{F}[V]_{\chi}^{G}$ for suitable linear characters $\chi: G \longrightarrow \mathbb{F}^{\times}$. In the case $\mathbb{F}=\mathbb{C}, \mathrm{R}$. P. Stanley made an extensive study of relative invariants of reflection groups in [9]. There is little trouble in carrying his results over to the nonmodular case (see, e.g., [5]). An analagous treatment of these results in a characteristic free way encompasing the modular case, though no more difficult, appears to be lacking in the literature, so we first provide one. We make no claim of originality for these results: In the nonmodular case these results are all well known, but often the nonmodular proofs do not directly translate to a characteristic free setting and need to be replaced by other arguments. This is particularly true of proofs using either the transfer homomorphism or Molien's Theorem. Once accomplished, we apply these results back to the original problem concerning the Cohen-Macaulay property. Since the Cohen-Macaulay property is not an issue in the nonmodular case, this would seem to be a new way to verify that a modular ring of invariants is Cohen-Macaulay.

I would like to thank V. Reiner, D. Stanton, and P. Webb for a number of interesting conversations concerning [3] which led to the results presented here, and J. Hartmann and A. Shepler for critical readings of this manuscript. As a general reference for invariant theory and unexplained notations, we refer to [5].

\section{RElative inVARIANTS AND EIGENSPACES}

Throughout this section $\mathbb{F}$ denotes a fixed field of characteristic $p$ which may be arbitrary and $G$ denotes a finite group. In addition we fix a faithful representation $\rho: G \hookrightarrow \mathrm{GL}(n, \mathbb{F})$ and a linear character $\chi: G \longrightarrow \mathbb{F}^{\times}$. The action of $G$ on the vector space $V=\mathbb{F}^{n}$ extends to the algebra $\mathbb{F}[V]$ of polynomial function $2^{2}$ on $V$ in the obvious way, viz., $(g f)(v)=f\left(g^{-1}(v)\right)$ for $g \in G$ and $f \in \mathbb{F}[V]$. There is then the ring of invariants

$$
\mathbb{F}[V]^{G}=\{f \in \mathbb{F}[V] \mid g f=f \forall g \in G\}
$$

and the module of relative invariants

$$
\mathbb{F}[V]_{\chi}^{G}=\{f \in \mathbb{F}[V] \mid g f=\chi(g) \cdot f \forall g \in G\} .
$$

\footnotetext{
${ }^{1}$ This was certainly an oversight in the course of writing $[5$.

${ }^{2}$ For readers not comfortable with the use of functions, take $\mathbb{F}[V]$ to be the symmetric algebra $S\left(V^{*}\right)$ on the vector space $V^{*}$ dual to $V$.
} 
The relation between the $\chi$-relative invariants and the invariants of the subgroup $H=\operatorname{ker}(\chi)$ are encorporated in the next lemma.

Lemma 1.1. Let $\rho: G \hookrightarrow \mathrm{GL}(n, \mathbb{F})$ be a faithful representation of a finite group over the field $\mathbb{F}, \chi: G \longrightarrow \mathbb{F}^{\times}$a linear character, and $H=\operatorname{ker}(\chi)$. Then $G / H$ is cyclic. If $g_{\chi} \in G$ generates $G / H$, then a form $f \in \mathbb{F}[V]$ belongs to $\mathbb{F}[V]_{\chi}^{G}$ if and only if

(i) $f \in \mathbb{F}[V]^{H}$, and

(ii) $g_{\chi} f=\chi\left(g_{\chi}\right) \cdot f$.

Proof. The group $G / H$ is cyclic since the finite subgroups of $\mathbb{F}^{\times}$are cyclic. Conditions (i) and (ii) are then immediate consequences of the definition of $\mathbb{F}[V]_{\chi}^{G}$. To prove the converse, note that $1, g_{\chi}, \ldots, g_{\chi}^{m-1}$ is a transversal for $H$ in $G$ if $m=|G: H|$. Let $f \in \mathbb{F}[V]^{H}$. If $u \in G$ is arbitrary, then we may write $u=g_{\chi}^{i} \cdot h$ for suitable $h \in H$ and $0 \leq i \leq m-1$. Then $\chi(u)=\chi\left(g_{\chi}\right)^{i}$, so (i) and (ii) imply

$$
u f=\left(g_{\chi}^{i} \cdot h\right)(f)=g_{\chi}^{i}(h f)=g_{\chi}^{i}(f)=\chi\left(g_{\chi}\right)^{i} \cdot f=\chi(u) \cdot f
$$

as required.

The connection between the relative invariants $\mathbb{F}[V]_{\chi}^{G}$ and the Cohen-Macaulay property of $\mathbb{F}[V]^{H}$, where $H=\operatorname{ker}(\chi)$, is provided by the decomposition of $\mathbb{F}[V]^{G}$ modules

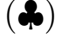

$$
\mathbb{F}[V]^{H}=\mathbb{F}[V]^{G} \oplus \mathbb{F}[V]_{\chi}^{G} \oplus \cdots \oplus \mathbb{F}[V]_{\chi^{m-1}}^{G} .
$$

By Lemma 1.1 the subspace $\mathbb{F}[V]_{\chi^{j}}^{G}$ for $j=0, \ldots, m-1$ may be viewed as the eigenspace corresponding to the eigenvalue $\chi\left(g_{\chi}\right)^{j}$ of the action of $g_{\chi}$ on $\mathbb{F}[V]^{H}$, where $g_{\chi} \cdot H \in G / H$ is a generator of the necessarily cyclic group $G / H$.

Central to the study of the relative invariants are the reflections in $G$. Recall that $s \in \mathrm{GL}(n, \mathbb{F})$ is called a reflection ${ }^{3}$ if it is not the identity, has finite order, and leaves a codimension one linear subspace $H_{s} \subsetneq V$ fixed. The subspace $H_{s}$ is called the reflecting hyperplane of $s$ : It is the eigenspace of $s$ corresponding to the eigenvalue 1 . The characteristic polynomial $p_{s}(t)$ of $s$ therefore has 1 as a root of multiplicity $n$ or $n-1$ and hence splits into a product of linear factors, viz., $p_{s}(t)=(t-1)^{n-1}\left(t-\lambda_{s}\right)$. The reflection $s$ is semisimple if and only if $\lambda_{s} \neq 1$. If $\lambda_{s}=1$, then the characteristic of the field $\mathbb{F}$ is $p \neq 0, s$ has order $p$, and is called a transvection. The following is then immediate from the fact that $\mathbb{F}^{\times}$contains no elements of order $p$.

Lemma 1.2. Let $\rho: G \hookrightarrow \operatorname{GL}(n, \mathbb{F})$ be a faithful representation of a finite group over the field $\mathbb{F}$ and $\chi: G \longrightarrow \mathbb{F}^{\times}$a linear character. Then $\operatorname{ker}(\chi)$ contains all the transvections in $G$.

\section{FREENESS OF MODULES OF RELATIVE INVARIANTS}

We are concerned with determining conditions under which $\mathbb{F}[V]_{\chi}^{G}$ is a free $\mathbb{F}[V]^{G}$ module. If it is, then Galois theory tells us it must be a cyclic $\mathbb{F}[V]^{G}$-module.

\footnotetext{
${ }^{3}$ In this generality reflections are sometimes called pseudoreflections. For a more detailed discussion of these matters see, e.g., [5], Sections 7.1 and 8.2.
} 
Lemma 2.1. Let $\rho: G \hookrightarrow \mathrm{GL}(n, \mathbb{F})$ be a faithful representation of a finite group over the field $\mathbb{F}$ and $\chi: G \longrightarrow \mathbb{F}^{\times}$a linear character. Then $\mathbb{F}[V]_{\chi}^{G}$ has rank one as an $\mathbb{F}[V]^{G}$-module $\rrbracket^{4}$ so if it is free, it is cyclic.

Proof. Set $H=\operatorname{ker}(\chi)$. The field extension $\mathbb{F}(V)^{G} \hookrightarrow \mathbb{F}(V)^{H}$ is Galois with Galois group $G / H$, which is a cyclic group of order $m$ generated by an element $g_{\chi} \cdot H \in$ $G / H$ such that $\chi\left(g_{\chi}\right) \in \mathbb{F}^{\times}$is a primitive $m$-th root of unity. Therefore $\mathbb{F}(V)^{H}$ affords the regular representation of $G / H \cong \mathbb{Z} / m$ as a $\mathbb{F}(V)^{G}$-representation. The decomposition of fields of fractions corresponding to $(\mathbf{p})$ is

$$
\mathbb{F}(V)^{H}=\mathbb{F}(V)^{G} \oplus \mathbb{F}(V)_{\chi}^{G} \oplus \cdots \oplus \mathbb{F}(V)_{\chi^{m-1}}^{G} .
$$

The subspace $\mathbb{F}(V)_{\chi^{j}}^{G}$ is a 1-dimensional $\mathbb{F}(V)^{G}$-subspace corresponding to the eigenvalue $\chi\left(g_{\chi}\right)^{j}$ of the action of $g_{\chi}$ on $\mathbb{F}(V)^{H}$. Since $\mathbb{F}(V)^{G} \otimes_{\mathbb{F}[V]^{G}} \mathbb{F}[V]_{\chi^{j}}^{G}$ is just $\mathbb{F}(V)_{\chi^{j}}^{G}$, the result follows.

There is even a reasonable candidate for a generator of $\mathbb{F}[V]_{\chi}^{G}$ if it is free as an $\mathbb{F}[V]^{G}$-module. To describe it we fix some more notations.

Notation. Let $s(G)$ denote the set of reflections in $G$ and $\mathcal{H}(G)=\left\{H_{s} \mid s \in s(G)\right\}$ the set of their reflecting hyperplanes. For $s \in s(G)$ choose a linear form $\ell_{s} \in$ $V^{*}=\mathbb{F}[V]_{1}$ such that $\operatorname{ker}\left(\ell_{s}\right)=H_{s}$. The form $\ell_{s}$ is well defined up to a nonzero constant. Since indeterminancy up to a nonzero scalar occurs often in the sequel, we introduce the notation $f^{\prime} \stackrel{\circ}{=} f^{\prime \prime}$ to indicate that two forms $f^{\prime}$ and $f^{\prime \prime}$ are nonzero multiples of each other.

If $U \in \mathcal{H}(G)$ is a reflecting hyperplane of $G$, we denote by $G_{U}$ the pointwise stabilizer of $U$ in $G$. This is the subgroup of $G$ consisting of all the reflections in $G$ with $U$ as a reflecting hyperplane together with $1 \in G$. For any finite group $G$ it has been shown by J. Hartmann and A. Shepler that $\mathbb{F}[V]^{G_{U}}$ is always a polynomial algebra (see [1]). The transvections in $G_{U}$ together with 1 compose the normal subgroup $\operatorname{ker}(\operatorname{det})$, where det $: G_{U} \longrightarrow \mathbb{F}^{\times}$is the restriction of the determinant to $G_{U}$. This is the unique $p$-Sylow subgroup $\operatorname{Syl}_{p}\left(G_{U}\right)$ of $G_{U}$ and is an elementary abelian $p$-group loc. cit. The quotient $G_{U} / \operatorname{Syl}_{p}\left(G_{U}\right)$ is a cyclic group. Let the order of this cyclic group be $m_{U}$ and choose $s_{U} \in G_{U}$ so that it projects to a generator of $G_{U} / \operatorname{Syl}_{p}\left(G_{U}\right)$. Then $\operatorname{det}\left(s_{U}\right) \in \mathbb{F}^{\times}$is a primitive $m_{U}$-th root of unity and $s_{U}$ is semisimple of order $m_{U}$.

Notation. For each $U \in \mathcal{H}(G)$ choose $a_{U} \in \mathbb{N}_{0}$ minimal such that $\chi\left(s_{u}\right)=\operatorname{det}\left(s_{u}\right)^{a_{u}}$ and introduce the form

$$
L_{\chi}=\prod_{U \in \mathcal{H}(G)} \ell_{s_{U}}^{a_{U}} \in \mathbb{F}[V]
$$

The form $L_{\chi}$, if it is $\chi$-relative invariant, is the only choice for a genarator of $\mathbb{F}[V]_{\chi}^{G}$ as an $\mathbb{F}[V]^{G}$-module if it is free. To see this we show that $L_{\chi}$ divides every $\chi$-relative invariant. For this we require two lemmas from 8 .

\footnotetext{
${ }^{4}$ By the rank of an $\mathbb{F}[V]^{G}$-module $M$ we mean the dimension of $F F\left(\mathbb{F}[V]^{G}\right) \otimes_{\mathbb{F}[V]^{G}} M$ as a vector space over the field of fractions $\operatorname{FF}\left(\mathbb{F}[V]^{G}\right)=\mathbb{F}(V)^{G}$, where $\mathbb{F}(V)$ is the field of rational functions on $V$.
} 
Lemma 2.2 (T. A. Springer $\left[\right.$, 4.2.4). Let $\ell \in \mathbb{F}[V]_{1}$ be a linear form and $s \in$ $\operatorname{GL}(n, \mathbb{F})$ a reflection. Suppose that $s(\ell)=\alpha \cdot \ell$ for some $\alpha \in \mathbb{F}^{\times}$. Then either

(i) $\alpha=1$, or

(ii) $\alpha=\operatorname{det}(s)$ and $\ell \stackrel{\circ}{=} \ell_{s}$.

Proof. For any form $f \in \mathbb{F}[V]$ the difference $s f-f$ is divisible by $\ell_{s}$, and we denote by $\Delta_{s}(f)$ the quotient 5 By [5], Lemma 7.1.5, $\Delta_{s}\left(\ell_{s}\right)=\lambda_{s}-1$, so

$$
\left(\lambda_{s}-1\right) \cdot \ell_{s}=\Delta_{s}(\ell) \cdot \ell_{s}=s(\ell)-\ell=(\alpha-1) \ell .
$$

If $\alpha \neq 1$, then this equation says that $\lambda_{s} \neq 1$ and $\ell$ and $\ell_{s}$ are proportional. The result then follows from the fact that in the notations agreed upon $\lambda_{s}=\operatorname{det}(s)$.

Lemma 2.3 (T. A. Springer [8], 4.3.3). Let $\ell_{1}, \ldots, \ell_{m} \in \mathbb{F}[V]_{1}$ be linear forms and $s \in \mathrm{GL}(n, \mathbb{F})$ a reflection. Suppose there are constants $\alpha_{1}, \ldots, \alpha_{m} \in \mathbb{F}^{\times}$such that

$$
s\left(\ell_{i}\right)= \begin{cases}\alpha_{i} \cdot \ell_{i+1} & \text { for } 1 \leq i \leq m-1, \\ \alpha_{m} \cdot \ell_{1} & \text { for } i=m .\end{cases}
$$

If none of $\ell_{1}, \ldots, \ell_{m}$ is a multiple of $\ell_{s}$, then the product $\alpha_{1} \cdots \alpha_{m}$ equals $1 \in \mathbb{F}^{\times}$, and therefore the form $L=\ell_{1} \cdots \ell_{m}$ of degree $m$ is s-invariant.

Proof. Clearly $s(L)=\alpha_{1} \cdots \alpha_{m} \cdot L$. Also from the hypotheses it follows that $s^{m}\left(\ell_{1}\right)=\alpha_{1} \cdots \alpha_{m} \cdot \ell_{1}$. If $s^{m} \neq 1$, then it is a reflection with reflecting hyperplane $\operatorname{ker}\left(\ell_{s}\right)$. Since $\ell_{1} \neq \ell_{s}$, it follows from Lemma 2.2 that $\alpha_{1} \cdots \alpha_{m}$ must equal 1. On the other hand, if $s^{m}=1$, then we would have $\ell_{1}=s^{m}\left(\ell_{1}\right)=\alpha_{1} \cdots \alpha_{m} \cdot \ell_{1}$, so again $\alpha_{1} \cdots \alpha_{m}=1$.

Lemma 2.4. Let $\rho: G \hookrightarrow \operatorname{GL}(n, \mathbb{F})$ be a faithful representation of a finite group over the field $\mathbb{F}$ and $\chi: G \longrightarrow \mathbb{F}^{\times}$a linear character. If $U \in \mathcal{H}(G)$ and $f \in \mathbb{F}[V]_{\chi}^{G}$, then $\ell_{s_{U}}^{a_{U}}$ divides $f$.

Proof. With no loss of generality we suppose $a_{U}>0$ in which case $s_{U}$ is a semisimple reflection since $\chi\left(s_{U}\right) \neq 1$. Choose a basis $u_{1}, \ldots, u_{n-1}$ for $U$ and extend it to a basis $u_{1}, \ldots, u_{n-1}, u_{n}$ for $V$ by selecting $u_{n}$ to be an eigenvector corresponding to the eigenvalue $\operatorname{det}\left(s_{U}\right) \neq 1 \in \mathbb{F}^{\times}$of $s_{U}$. Let $z_{1}, \ldots, z_{n} \in V^{*}=\mathbb{F}[V]_{1}$ be the dual basis for the space of linear forms so $\ell_{s_{U}} \stackrel{\circ}{=} z_{n}$. The action of $s_{U}$ on $\mathbb{F}[V]$ sends a monomial in $z_{1}, \ldots, z_{n}$ to a multiple of itself; so a form $f$ is $\left.\chi\right|_{G_{U}}$-relative invariant if and only if it is a sum of $\left.\chi\right|_{G_{U}}$-relative invariant monomials. For such a monomial $z^{E}=z_{1}^{e_{1}} \cdots z_{n}^{e_{n}}$, we have by Lemma 2.2,

$$
\chi\left(s_{U}\right) \cdot z^{E}=s_{U}\left(z^{E}\right)=\operatorname{det}\left(s_{U}\right)^{e_{n}} \cdot z^{E} .
$$

Since $a_{U}$ is the smallest natural number such that $\chi\left(s_{U}\right)=\operatorname{det}\left(s_{U}\right)^{a_{U}}$, we must have $a_{U}$ divides $e_{n}$, and the result follows.

Proposition 2.5. Let $\rho: G \hookrightarrow \mathrm{GL}(n, \mathbb{F})$ be a faithful representation of a finite group over the field $\mathbb{F}$ and $\chi: G \longrightarrow \mathbb{F}^{\times}$a linear character. Then $L_{\chi}$ divides any element $f \in \mathbb{F}[V]_{\chi}^{G}$.

Proof. This follows from Lemma 2.4 and the fact that the forms $\ell_{s_{U}}$ are pairwise relatively prime as $U$ ranges over the distinct elements of $\mathcal{H}(G)$.

\footnotetext{
${ }^{5}$ See [5], Section 7.1, and the references there for more about the operators $\Delta_{s}$.
} 
To complete the discussion we need to decide if $L_{\chi}$ is a $\chi$-relative invariant. To this end rewrite $L_{\chi} \in \mathbb{F}[V]$ in the form

$$
L_{\chi}=\ell_{s_{U^{\prime}}}^{a_{U^{\prime}}} \cdot \prod_{\substack{U^{\prime \prime} \in \mathcal{H}(G) \\ U^{\prime \prime} \neq U^{\prime}}} \ell_{s_{U^{\prime \prime}}}^{a_{U^{\prime \prime}}}
$$

where $U^{\prime} \in \mathcal{H}(G)$ is a fixed reflecting hyperplane of $G$. Since $\ell_{s_{U^{\prime \prime}}} \neq \ell_{s_{U^{\prime}}}$ if $U^{\prime \prime} \neq U^{\prime}$, it follows from Lemma 2.3 that the product

$$
\prod_{\substack{U^{\prime \prime} \in \mathcal{H}(G) \\ U^{\prime \prime} \neq U^{\prime}}} \ell_{s_{U^{\prime \prime}}}^{a_{U^{\prime \prime}}}
$$

is $s_{U^{\prime}}$-invariant. For the other factor of $L_{\chi}$ we have

$$
s_{U^{\prime}}\left(\ell_{s_{U^{\prime}}}^{a_{U^{\prime}}}\right)=s_{U^{\prime}}\left(\ell_{s_{U^{\prime}}}\right)^{a_{U^{\prime}}}=\left(\operatorname{det}\left(s_{U^{\prime}}\right) \cdot \ell_{s_{U^{\prime}}}\right)^{a_{U^{\prime}}}=\operatorname{det}\left(s_{U^{\prime}}\right)^{a_{U^{\prime}}} \cdot \ell_{s_{U^{\prime}}}^{a_{U^{\prime}}}=\chi\left(s_{U^{\prime}}\right) \cdot \ell_{s_{U^{\prime}}}^{a_{U^{\prime}}},
$$

so $\ell_{S_{U^{\prime}}}^{a_{U^{\prime}}}$ is a $\left.\chi\right|_{G_{U^{\prime}}}$-relative invariant. Therefore $L_{\chi} \in \mathbb{F}[V]_{\chi_{U_{U^{\prime}}}}^{G_{U^{\prime}}}$. If $G$ is generated by reflections, then this is enough to deduce the following result.

Proposition 2.6. Let $\rho: G \hookrightarrow \operatorname{GL}(n, \mathbb{F})$ be a faithful representation of a finite group over the field $\mathbb{F}$ and $\chi: G \longrightarrow \mathbb{F}^{\times}$a linear character. Suppose that $G$ is generated by $s(G)$. Then the form

$$
L_{\chi}=\prod_{U \in \mathcal{H}(G)} \ell_{s_{U}}^{a_{U}} \in \mathbb{F}[V]
$$

is a $\chi$-relative invariant, i.e., $L_{\chi} \in \mathbb{F}[V]_{\chi}^{G}$.

Proof. From the preceding discussion we have $s\left(L_{\chi}\right)=\chi(s) \cdot L_{\chi}$ for any $s \in s(G)$. If $g \in G$ is arbitrary we may write $g=s_{1} \cdots s_{k}$ for suitable $s_{1}, \ldots, s_{k} \in s(G)$, whence

$g\left(L_{\chi}\right)=\left(s_{1} \cdots s_{k}\right)\left(L_{\chi}\right)=\left(s_{1}\left(s_{2} \cdots\left(s_{k}\left(L_{\chi}\right)\right) \cdots\right)=\chi\left(s_{1}\right) \cdots \chi\left(s_{k}\right) \cdot L_{\chi}=\chi(g) \cdot L_{\chi}\right.$,

establishing that $L_{\chi}$ is $\chi$-relative invariant.

Combining Propositions 2.5 and 2.6 leads us to the modular analogue of the main result of R. P. Stanley on relative invariants of reflection groups (see [9], Theorem 3.1). Before formulating this result we remind the reader that a group generated by reflections need not have polynomial invariants in the modular case (see, e.g., [5], §7.4, Example 4).

Theorem 2.7. Let $\rho: G \hookrightarrow \operatorname{GL}(n, \mathbb{F})$ be a faithful representation of a finite group over the field $\mathbb{F}$ and $\chi: G \longrightarrow \mathbb{F}^{\times}$a linear character. Suppose that $G$ is generated by $s(G)$. Then $\mathbb{F}[V]_{\chi}^{G}$ is a free module over $\mathbb{F}[V]^{G}$ on the single generator $L_{\chi}$.

Proof. Proposition 2.5 implies that every element of $f \in \mathbb{F}[V]_{\chi}^{G}$ is divisible by $L_{\chi}$. By Proposition [2.6 $L_{\chi} \in \mathbb{F}[V]_{\chi}^{G}$ since $G$ is generated by reflections. Therefore $f / L_{\chi} \in \mathbb{F}[V]^{G}$ and the map $\mathbb{F}[V]^{G} \longrightarrow \mathbb{F}[V]_{\chi}^{G}$ defined by $h \mapsto h \cdot L_{\chi}$ is an isomorphism of degree $\operatorname{deg}\left(L_{\chi}\right)$, as required. 


\section{Some Rings of invariants that ARE COHEN-Macaulay}

To begin this section we reformulate the problem described in the introduction so as to bring the results on relative invariants of the previous section to bear.

Start with a faithful representation $\rho: G \hookrightarrow \operatorname{GL}(n, \mathbb{F})$ of a finite group $G$ and a linear character $\chi: G \longrightarrow \mathbb{F}^{\times}$. Set $H=\operatorname{ker}(\chi)$. Then $H \triangleleft G$ is a normal subgroup of $G$ and $\operatorname{Im}(\chi) \leq \mathbb{F}^{\times}$is a finite cyclic group of order prime to the characteristic of $\mathbb{F}$. Let $\operatorname{Im}(\chi)$ have order $m$ so it is generated by a primitive $m$-th root of unity $\xi \in \mathbb{F}^{\times}$. Let $g_{\chi} \in G$ project in $G / H$ to a generator such that $\chi\left(g_{\chi}\right)=\xi$. The group $G / H$ acts on $\mathbb{F}[V]^{H}$ and decomposes it into eigenspaces of $g_{\chi}$. These are just the modules of relative invariants of the linear characters $\chi^{i}$ for $i=0, \ldots, m-1$ (see Lemma 1.1), viz.,

$$
\mathbb{F}[V]^{H}=\mathbb{F}[V]^{G} \oplus \mathbb{F}[V]_{\chi}^{G} \oplus \cdots \oplus \mathbb{F}[V]_{\chi^{m-1}}^{G} .
$$

Choose a system of parameters $f_{1}, \ldots, f_{n} \in \mathbb{F}[V]^{G}$. Then restricting the module structure from $\mathbb{F}[V]^{G}$ to $\mathbb{F}\left[f_{1}, \ldots, f_{n}\right]$ we see that $\mathbb{F}[V]^{H}$ is Cohen-Macaulay if and only if the modules of relative invariants $\mathbb{F}[V]_{\chi^{i}}^{G}$ are free $\mathbb{F}\left[f_{1}, \ldots, f_{n}\right]$-modules for $i=0, \ldots, m-1$. We collect this conclusion for further use.

Lemma 3.1. Suppose $\rho: G \hookrightarrow \operatorname{GL}(n, \mathbb{F})$ is a faithful representation of a finite group $G$ over the field $\mathbb{F}$ and $\chi: G \longrightarrow \mathbb{F}^{\times}$is a linear character of $G$ defined over $\mathbb{F}$. Set $H=\operatorname{ker}(\chi)$ and let $m=|G: H|$. Choose a system of parameters $f_{1}, \ldots, f_{n} \in \mathbb{F}[V]^{G}$. Then as $\mathbb{F}\left[f_{1}, \ldots, f_{n}\right]$-modules

$$
\mathbb{F}[V]^{H}=\mathbb{F}[V]^{G} \oplus \mathbb{F}[V]_{\chi}^{G} \oplus \cdots \oplus \mathbb{F}[V]_{\chi}^{G-1},
$$

so $\mathbb{F}[V]^{H}$ is Cohen-Macaulay if and only if the $\mathbb{F}\left[f_{1}, \ldots, f_{n}\right]$-modules $\mathbb{F}[V]_{\chi^{i}}^{G}, i=$ $0, \ldots, m$, are free.

Remark. Let $\rho: G \hookrightarrow \operatorname{GL}(n, \mathbb{F})$ be a representation of a finite group and $H \leq G$ a subgroup with index $|G: H|$ relative prime to the characteristic of $\mathbb{F}$. A simple argument using the relative transfer $\operatorname{Tr}_{H}^{G}$ shows $\mathbb{F}[V]^{H}$ is Cohen-Macaulay implies that $\mathbb{F}[V]^{G}$ is also (see, e.g., the proof of Theorem 6.7.8 in [5]) Cohen-Macaulay. Proposition 3.1 is a sort of refinement of this.

Theorem 3.2. Suppose $\rho: G \hookrightarrow \operatorname{GL}(n, \mathbb{F})$ is a faithful representation of a finite group $G$ over the field $\mathbb{F}$ and $\chi: G \longrightarrow \mathbb{F}^{\times}$is a linear character of $G$ defined over $\mathbb{F}$. Set $H=\operatorname{ker}(\chi)$ and suppose that $\mathbb{F}[V]^{G}$ is a polynomial algebra. Then $\mathbb{F}[V]^{H}$ is Cohen-Macaulay.

Proof. Since $\mathbb{F}[V]^{G}$ is a polynomial algebra, $G$ is generated by reflections (see [4]). By Theorem 2.7 the modules of relative invariants $\mathbb{F}[V]_{\chi^{i}}^{G}$ are free $\mathbb{F}[V]^{G}$-modules for $i=0, \ldots,|G H|-1$, so the conclusion follows from Lemma 3.1 .

\section{Subgroups stabilizing an Eigenspace}

Definition. For a representation $\rho: G \hookrightarrow \operatorname{GL}(n, \mathbb{F})$ of a finite group over the field $\mathbb{F}$ and a subspace $U \leq V=\mathbb{F}^{n}$, denote by $G_{\langle U\rangle}$ the subgroup of $G$ consisting of elements having $U$ as an eigenspace. If $U$ is 1-dimensional and $0 \neq x \in U$, then we write $G_{\langle x\rangle}$ for $G_{\langle U\rangle}$.

Theorem 4.1. Let $\rho: G \hookrightarrow \operatorname{GL}(n, \mathbb{F})$ be a representation of a finite group over the field $\mathbb{F}$ and $U \leq V=\mathbb{F}^{n}$ a subspace. If $\mathbb{F}[V]^{G}$ is Cohen-Macaulay, then so is $\mathbb{F}[V]^{G\langle U\rangle}$. 
Proof. We start with the case where $\operatorname{dim}_{\mathbb{F}}(U)=1$ and $0 \neq x \in U$. Since $x$ is an eigenvector of every element $g \in G_{\langle x\rangle}$, we may define a linear character $\lambda_{x}: G_{\langle x\rangle} \longrightarrow \mathbb{F}^{\times}$by the requirement that $\lambda(g) \cdot x=g(x)$. Then $\operatorname{ker}\left(\lambda_{x}\right)=G_{x}$ is the pointwise stabilizer of the line spanned by $x$. If $\mathbb{F}[V]^{G}$ is Cohen-Macaulay, then so is $\mathbb{F}[V]^{G_{x}}$ by [6], Theorem 2.1. The group $G_{\langle x\rangle} / G_{x}$ is isomorphic to a subgroup of $\mathbb{F}^{\times}$and so has order relative prime to the characteristic of $\mathbb{F}$. Hence the relative transfer

$$
\operatorname{Tr}_{G_{x}}^{G_{\langle x\rangle}}: \mathbb{F}[V]^{G_{\langle x\rangle}} \longrightarrow \mathbb{F}[V]^{G_{x}}
$$

is a splitting for the inclusion

$$
\mathbb{F}[V]^{G_{\langle x\rangle} \hookrightarrow \mathbb{F}[V]^{G_{x}},}
$$

and therefore (cf. [5], the proof of Theorem 6.7.8) $\mathbb{F}[V]^{G_{\langle x\rangle}}$ is Cohen-Macaulay.

We may therefore proceed by induction, assuming that $\operatorname{dim}_{\mathbb{F}}(U)=k$ and the result is established for all subspaces of dimension strictly smaller than $k$. Choose a nonzero vector $x \in U$ and decompose $U$ into the span of $x$ and a subspace $W<U$ of dimension $k-1$. Then $\mathbb{F}[V]^{G\langle W\rangle}$ is Cohen-Macaulay by the induction hypothesis. Since $G_{\langle U\rangle}=\left(G_{\langle W\rangle}\right)_{\langle x\rangle}$, a second application of the induction hypothesis completes the proof.

Other properties of $\mathbb{F}[V]^{G}$ inherited by $\mathbb{F}[V]^{G\langle U\rangle}$ are studied in $[7$.

\section{REFERENCES}

[1] J. Hartmann and A. Shepler, Jacobians of Reflection Groups over Finite Fields, Preprint, 2004.

[2] V. Reiner, Free Modules of Relative Invariants of Finite Groups, Studies in Applied Math. 81 (1989), 181-184. MR1016588 (90k:20018)

[3] V. Reiner, D. Stanton, and P. Webb, Springer's Regular Elements over Arbitrary Fields, Preprint, Uni. of Minn., 2004.

[4] J.-P. Serre, Groupes finis d'automorphismes d'anneaux locaux réguliers, Colloq. d'Alg. Éc. Norm. Sup. de Jeunes Filles, Paris, 8-01-8-11, 1967. MR0234953 (38:3267)

[5] L. Smith, Polynomial Invariants of Finite Groups, A.K. Peters, Ltd., Wellesley, MA, 1995, second printing 1997. MR1328644 (96f:13008)

[6] L. Smith, Lannes T-Functor and the Invariants of Pointwise Stabilizers, Forum. Math. 12 (2000), 461-476. MR1763901 (2001e:13009)

[7] L. Smith, Reflections on Reflection Groups (to appear).

[8] T. A. Springer, Invariant Theory, Lecture Notes in Math. 585, Springer-Verlag, Berlin, 1977. MR0447428 (56:5740)

[9] R. P. Stanley, Relative invariants of Finite Groups generated by Pseudoreflections, J. of Algebra 49 (1977), 134-148. MR0460484 (57:477)

Mathematisches Institut, Bunsenstrasse 3-5, D 37073 Göttingen, Federal Republic of GERMANY

E-mail address: larry@uni-math.gwdg.de 